\title{
Application of Differential Geometry in Magnetic Levitation Control
}

\author{
Hengkun Liu*, Yungang Li and Hu Cheng \\ Research center of Maglev, National University of Defense Technology, Changsha, Hunan, China
}

Received: 11 Sep. 2013, Revised: 9 Dec. 2013, Accepted: 10 Dec. 2013

Published online: 1 Sep. 2014

\begin{abstract}
To enhance the reliability of levitation, an especial installation called joint-structure is applied to the maglev vehicle. Due to the joint-structure, each suspension point of the maglev vehicle is regulated by two independent controllers. When one controller of the suspension point breaks down, the system can still be stably suspended by the other controller. However, there is strong force-coupling between the two controllers for the joint-structure, which makes the controller designed based on totally separated suspension point unsuccessful in application. To realize stable suspension for joint-structure, diffenrential geometry method is introduced to obtain a globally decoupling and linearized model for the system. Then the control parameters are designed by pole assignment. Experimental and simulation results validate the effectiveness of the proposed control algorithm.
\end{abstract}

Keywords: Maglev vehicles, Nonlinear decoupling control, Differential geometry

\section{INTRODUCTION}

Maglev technology has been widely utilized to different applications[1], among which maglev vehicles rapidly develops since its presentation. To enhance the reliability of maglev vehicles, joint-structure is introduced, and each joint-structure is regulated by two controllers. If one controller breaks down, the other controller can still guarantee the stability of the system, and the stability of the vehicle accordingly. As the two controllers for the same joint-structure is strongly coupled together, decoupling control is the key to the stability of the joint-structure. There are quite a lot of decoupling control strategies, such as inversion matrix method [2,3], relative amplification coefficient matching method[4], diagonal dominance approach[5], state feedback control[6], adaptive decoupling control[7,8], intelligent decoupling control $[9,10]$, nonlinear and robust decoupling control[11,12], and so on. Based on the characteristics of the maglev system and the experimental setup, this article uses differential geometry method to realize the decoupling and the stability of the joint-structure.

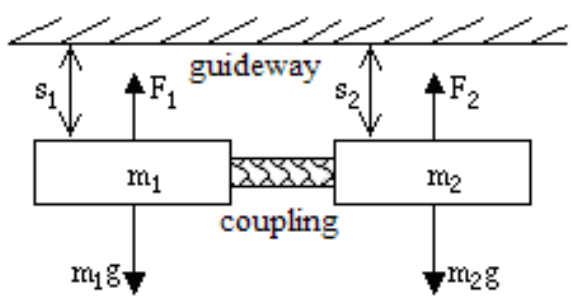

Fig. 1: Sketch of joint-structure

\section{MODELING}

Ignoring the elasticity of the guideway and the effect of the air-spring on the system performance, the sketch of the joint-structure can be presented by Fig. 1 [13].

The definition of the symbols in Fig. 1 and the symbols which will be used in the following are presented as below.

$s_{01}=s_{02}=s_{0}$ is the expected suspension gap, $s_{1}$ and $s_{2}$ is the measured suspension gap, $m_{1}=m_{2}=m$ is mass of the suspension object, $g$ is the Gravity acceleration, $F_{1}$ and $F_{2}$ is the electromagnetic force, $u_{1}$ and $u_{2}$ is the control voltage, $i_{1}$ and $i_{2}$ is the current in the coil, $N_{1}=N_{2}=N$ is the turns of the coil, $R_{1}=R_{2}=R$ is the resistance of the coil, $A_{1}=A_{2}=A$ is the pole area of

\footnotetext{
* Corresponding author e-mail: liberry@ sina.com
} 
a single side of the electromagnet, $L_{1}$ and $L_{2}$ is the inductance of the coil, $\mu_{0}$ is the permeability of vacuum, $k_{r}$ is the rigid coefficient between two sides.

Suppose the magnetomotive force of the magnetic field concentrates in the air gap, the mathematical model of the system shown in Fig. 1 can be obtained as[13],

$$
\begin{aligned}
& \left\{\begin{array}{c}
u_{1}=R i_{1}+\frac{\mu_{0} N^{2} A}{2 s_{1}} \frac{d i_{1}}{d t}-\frac{\mu_{0} N^{2} A i_{1}}{2 s_{1}^{2}} \frac{d s_{1}}{d t} \\
m \ddot{s}_{1}=-F_{1}\left(i_{1}, s_{1}\right)+m g-k_{r}\left(s_{1}-s_{2}\right) \\
u_{2}=R i_{2}+\frac{\mu_{0} N^{2} A}{2 s_{2}} \frac{d i_{2}}{d t}-\frac{\mu_{0} N^{2} A i_{2}}{2 s_{2}^{2}} \frac{d s_{2}}{d t} \\
m \ddot{s}_{2}=-F_{2}\left(i_{2}, s_{2}\right)+m g+k_{r}\left(s_{1}-s_{2}\right)
\end{array}\right. \\
& F_{i}\left(i_{i}, s_{i}\right)=\left(\mu_{0} N^{2} A / 4\right)\left(i_{i} / s_{i}\right)^{2}
\end{aligned}
$$

In (1), the first and the second equations are separately the electricity equation and mechanical equation for the suspension system on the left, while the third and the fourth equations are separately the electricity equation and mechanical equation for the suspension system on the right.

\section{ESSENTIAL THEORY OF DIFFERENTIAL GEOMETRY}

For the convenience of problem analysis, the decoupling control theory used in this paper is listed as following[14].

Definition 1: Let open sets, $U \subset R^{n}, x \subset U$, and given a smooth scalar function $\lambda(x)$ and a $n$-dimention vector field $f(x)$ in $U$. Then a new scalar function noted $L_{f} \lambda(x)$ is defined as following.

$$
L_{f} \lambda(x)=\sum_{i=1}^{n} \frac{\partial \lambda}{\partial x_{i}} f_{i}\left(x_{1}, x_{2}, \ldots, x_{n}\right)
$$

This new function $L_{f} \lambda(x)$ is called the lie-algebra of $\lambda(x)$ along $f(x)$. And this function can be calculated iteratively. For example, lie-algebra of $\lambda(x)$ sequentially along $f(x)$ and $g(x)$ is,

$$
L_{g} L_{f} \lambda(x)=\frac{\partial\left(L_{f} \lambda\right)}{\partial x} g(x)
$$

Or lie-algebra of $\lambda(x)$ along $f(x)$ for $k$ times is,

$$
L_{f}^{k} \lambda(x)=d\left(L_{f}^{k-1} \lambda\right) f(x)
$$

Definition 2: For the following multiple inputs and multiple outputs system,

$$
\begin{aligned}
& \dot{x}=f(x)+\sum_{i=1}^{m} g_{i}(x) u_{i} \\
& y_{j}=h_{j}(x), j \in \underline{m}
\end{aligned}
$$

Its relative order $r_{i}\left(x_{0}\right)$ is the lie algebra, which satisfies,

$$
L_{g_{j}} L_{f}^{r_{i}-1} h_{i}\left(x_{0}\right) \neq 0(j=1, \ldots, m)
$$

And for $k<r_{i}-1$, satisfies,

$$
L_{g_{j}} L_{f}^{k} h_{i}\left(x_{0}\right)=0(j=1, \ldots, m)
$$

On the basis of the two definitions, two theorems for decoupling of nonlinear systems are introduced. First, introduce the decoupling matrix nonsingular theorem.

Theorem 1: If a multiple variables nonlinear system has a relative order $r_{i}$ for $x_{0}$ to all $i \in \underline{m}$, its decoupling matrix $A(x)$ shown in (6) is nonsingular at $x_{0}$.

$$
\left[\begin{array}{ccc}
L_{g_{1}} L_{f}^{r_{1}-1} h_{1}(x) & \cdots & L_{g_{m}} L_{f}^{r_{1}-1} h_{1}(x) \\
L_{g_{1}} L_{f}^{r_{2}-1} h_{2}(x) & \cdots & L_{g_{m}} L_{f}^{r_{2}-1} h_{2}(x) \\
\vdots & \cdots & \vdots \\
L_{g_{1}} L_{f}^{r_{m}-1} h_{m}(x) & \cdots & L_{g_{m}} L_{f}^{r_{m}-1} h_{m}(x)
\end{array}\right]
$$

Then introduce theorem 2, which is utilized to explain the conditions for decoupling of the system, and how to realize decoupling for nonlinear coupled system.

Theorem 2: If nonlinear system has relative order at $x_{0}$, or saying decoupling matrix $A(x)$ is nonsingular at $x_{0}$, then the input-output decoupling problem at $x_{0}$ can be solved by a static state feedback, and one solution is the feedback defined by the following matrix,

$$
\begin{aligned}
& u(x)=\alpha(x)+\beta(x) v \\
& \alpha(x)=-A^{-1}(x) b(x) \\
& \beta(x)=A^{-1}(x)
\end{aligned}
$$

where,

$$
b(x)=\left[L_{f}^{r_{1}} h_{1}(x) \cdots L_{f}^{r_{m}} h_{m}(x)\right]^{T}
$$

If the conditions described in theorem 2 are satisfied, decoupling for nonlinear system can be realized by equations (7) and (8). The proposed nonlinear decoupling method for maglev system can be obtained based on the definitions and theorems introduced in this part.

\section{DECOUPLING AND LINEARIZATION OF THE SYSTEM}

For the convenience of description, introduce the following variables transform,

$$
\begin{gathered}
x_{1}=s_{1} x_{2}=y_{1} x_{3}=i_{1} \\
x_{4}=s_{2} x_{5}=y_{2} x_{6}=i_{2} \\
k=\mu_{0} N^{2} A / 4
\end{gathered}
$$


By the transform in (9), the model (1) can be changed as following,

$$
\begin{aligned}
& \left\{\begin{array}{l}
\dot{x}_{1}=x_{2} \\
\dot{x}_{2}=g-\frac{k x_{3}^{2}}{m x_{1}^{2}}-\frac{k_{r}\left(x_{1}-x_{4}\right)}{m} \\
\dot{x}_{3}=\frac{x_{2} x_{3}}{x_{1}}-\frac{R x_{1} x_{3}}{2 k}+\frac{x_{1}}{2 k} u_{1} \\
\dot{x}_{4}=x_{5} \\
\dot{x}_{5}=g-\frac{k x_{6}^{2}}{m x_{4}^{2}}+\frac{k_{r}\left(x_{1}-x_{4}\right)}{m} \\
\dot{x}_{6}=\frac{x_{5} x_{6}}{x_{4}}-\frac{R x_{4} x_{6}}{2 k}+\frac{x_{4}}{2 k} u_{2}
\end{array}\right. \\
& Y=\left[\begin{array}{llllll}
1 & 0 & 0 & 0 & 0 & 0 \\
0 & 0 & 0 & 1 & 0 & 0
\end{array}\right] X
\end{aligned}
$$

Compared with the standard form in (5), it can be known:

$$
\begin{aligned}
& f(x)=\left[\begin{array}{c}
x_{2} \\
g-k x_{3}^{2} / m x_{1}^{2}-k_{r}\left(x_{1}-x_{4}\right) / m \\
x_{2} x_{3} / x_{1}-R x_{1} x_{3} / 2 k \\
x_{5} \\
g-k x_{6}^{2} / m x_{4}^{2}+k_{r}\left(x_{1}-x_{4}\right) / m \\
x_{5} x_{6} / x_{4}-R x_{4} x_{6} / 2 k
\end{array}\right] \\
& g_{1}(x)=\left[\begin{array}{lllllll}
0 & 0 & x_{1} / 2 k & 0 & 0 & 0
\end{array}\right]^{T} \\
& g_{2}(x)=\left[\begin{array}{llllll}
0 & 0 & 0 & 0 & 0 & x_{4} / 2 k
\end{array}\right]^{T} \\
& y_{1}=h_{1}(x)=x_{1} \\
& y_{2}=h_{2}(x)=x_{4}
\end{aligned}
$$

First, the decoupling matrix of the system can be calculated according to (6) as,

$$
A(x)=\left[\begin{array}{cc}
-x_{3} / m x_{1} & 0 \\
0 & -x_{6} / m x_{4}
\end{array}\right]
$$

And the other expressions to calculated the control variables can also be obtained as,

$$
\begin{gathered}
b(x)=\left[\begin{array}{ll}
b_{1} b_{2}
\end{array}\right]^{T}= \\
{\left[\frac{R x_{3}^{2}}{m x_{1}}-\frac{k_{r}}{m}\left(x_{2}-x_{5}\right) \frac{R x_{6}^{2}}{m x_{4}}+\frac{k_{r}}{m}\left(x_{2}-x_{5}\right)\right]} \\
\alpha(x)=\left[\begin{array}{l}
R x_{3}-k_{r} x_{1}\left(x_{2}-x_{5}\right) / x_{3} \\
R x_{6}+k_{r} x_{4}\left(x_{2}-x_{5}\right) / x_{6}
\end{array}\right] \\
\beta(x)=\left[\begin{array}{cc}
-m x_{1} / x_{3} & 0 \\
0 & -m x_{4} / x_{6}
\end{array}\right]
\end{gathered}
$$

By equation (7), the decoupling control law can be calculated as,

$$
\begin{aligned}
{\left[\begin{array}{l}
u_{1} \\
u_{2}
\end{array}\right]=} & {\left[\begin{array}{l}
R x_{3}-k_{r} x_{1}\left(x_{2}-x_{5}\right) / x_{3} \\
R x_{6}+k_{r} x_{4}\left(x_{2}-x_{5}\right) / x_{6}
\end{array}\right] } \\
& +\left[\begin{array}{cc}
-m x_{1} / x_{3} & 0 \\
0 & -m x_{4} / x_{6}
\end{array}\right]\left[\begin{array}{l}
v_{1} \\
v_{2}
\end{array}\right]
\end{aligned}
$$

That's to say,

$$
\begin{aligned}
& u_{1}=R x_{3}-\frac{k_{r} x_{1}}{x_{3}}\left(x_{2}-x_{5}\right)-\frac{m x_{1}}{x_{3}} v_{1} \\
& u_{2}=R x_{6}+\frac{k_{r} x_{4}}{x_{6}}\left(x_{2}-x_{5}\right)-\frac{m x_{4}}{x_{6}} v_{2}
\end{aligned}
$$

Up to now, the system has been decoupled. To realize linearization of the decoupled system, the following variable transform is introduced,

$$
z_{1}=x_{1}, z_{2}=x_{2}
$$

$$
\begin{gathered}
z_{3}=\dot{z}_{2}=g-\frac{k x_{3}^{2}}{m x_{1}^{2}}-\frac{k_{r}\left(x_{1}-x_{4}\right)}{m} \\
z_{4}=x_{4}, z_{5}=x_{5}, \\
z_{6}=\dot{z}_{5}=g-\frac{k x_{6}^{2}}{m x_{4}^{2}}+\frac{k_{r}\left(x_{1}-x_{4}\right)}{m}
\end{gathered}
$$

It can be obtained by calculation that,

$\dot{z}_{3}=v_{1}, \dot{z}_{6}=v_{2}$

Now, the model for joint-structure has been decoupled and linearized, and the state space model after decoupling is,

$$
\dot{z}=A z+B v
$$

$$
y=C z
$$

where,

$$
z=\left[\begin{array}{llll}
z_{1} & z_{2} & \cdots & z_{6}
\end{array}\right]^{T}
$$

And the state matrix for the system is,

$$
\begin{aligned}
& A=\left[\begin{array}{llllll}
0 & 1 & 0 & 0 & 0 & 0 \\
0 & 0 & 1 & 0 & 0 & 0 \\
0 & 0 & 0 & 0 & 0 & 0 \\
0 & 0 & 0 & 0 & 1 & 0 \\
0 & 0 & 0 & 0 & 0 & 1 \\
0 & 0 & 0 & 0 & 0 & 0
\end{array}\right] \\
& B=\left[\begin{array}{llllll}
0 & 0 & 1 & 0 & 0 & 0 \\
0 & 0 & 0 & 0 & 0 & 1
\end{array}\right]^{T} \\
& C=\left[\begin{array}{llllll}
1 & 0 & 0 & 0 & 0 & 0 \\
0 & 0 & 0 & 1 & 0 & 0
\end{array}\right]
\end{aligned}
$$


From state space matrix $(32) \sim(34)$, the state space model for two separated subsystems can be obtained, and the state space for the first subsystem is,

$$
\begin{aligned}
& A_{1}=\left[\begin{array}{lll}
0 & 1 & 0 \\
0 & 0 & 1 \\
0 & 0 & 0
\end{array}\right] \\
& B_{1}=\left[\begin{array}{lll}
0 & 0 & 1
\end{array}\right]^{T} \\
& C_{1}=\left[\begin{array}{lll}
1 & 0 & 0
\end{array}\right]
\end{aligned}
$$

It can be seen from $(32) \sim(34)$ that, the state matrix of the second subsystem is the same to the first one. Thus only the controller design for the first subsystem is conducted.

\section{CONTROL ALGORITHM DESIGN}

In this section, control algorithm design refers to the controller design for subsystem $(35) \sim(37)$. For the convenience of implementation, state feedback control is utilized. Suppose the feedback control law is [15],

$$
v_{1}=k_{1}\left(z_{1}-z_{01}\right)+k_{2} z_{2}+k_{3} z_{3}
$$

And set the control goals of the system after feedback as, overshoot is less than $5 \%$, settling time is $0.1 s$. Then the dominant poles for the system is calculated as,

$$
\begin{aligned}
& s_{1}=-20+42.925 j \\
& s_{2}=-20-42.925 j
\end{aligned}
$$

Suppose the third pole of the system is,

$$
s_{3}=-200
$$

Then the characteristic equation for the system is,

$$
s^{3}+280 s^{2}+19358 s+6715420
$$

Substitute feedback control law (38) into the first subsystem after linearization $(35) \sim(37)$, and the characteristic equation for the closed loop system can be obtained as,

$$
s^{3}-k_{3} s^{2}-k_{2} s-k_{1}=0
$$

Comparing the characteristic equation after feedback control (43) with the expected characteristic equation (42), the control parameters can be chosen as,

$$
\begin{gathered}
k_{1}=-671542 \\
k_{2}=-19358 \\
k_{3}=-280
\end{gathered}
$$

Substitute (25) and (26) into (38), the control variable for the linear system can be calculated as,

$$
\begin{aligned}
& v_{1}=k_{1}\left(x_{1}-s_{0}\right)+k_{2} x_{2}+ \\
& k_{3}\left(g-\frac{k x_{3}^{2}}{x_{1}^{2}}-\frac{k_{r}\left(x_{1}-x_{4}\right)}{m}\right)
\end{aligned}
$$

Substitute control law for linear system (47) into the expression of control variable (23), the control variable of the first subsystem for the joint-structure can be obtained as,

$$
\begin{aligned}
& u_{1}=R x_{3}-\frac{k_{r} x_{1}}{x_{3}}\left(x_{2}-x_{5}\right) \\
& -\frac{m x_{1}}{x_{3}}\left[\begin{array}{l}
k_{1}\left(x_{1}-s_{0}\right)+k_{2} x_{2}+ \\
k_{3}\left(g-\frac{k x_{3}^{2}}{m x_{1}^{2}}-\frac{k_{r}\left(x_{1}-x_{4}\right)}{m}\right)
\end{array}\right]
\end{aligned}
$$

Similarly, the control variable for the second subsystem can be calculated as,

$$
\begin{aligned}
& u_{2}=R x_{6}+\frac{k_{r} x_{4}}{x_{6}}\left(x_{2}-x_{5}\right) \\
& -\frac{m x_{4}}{x_{6}}\left[\begin{array}{l}
k_{1}\left(x_{4}-s_{0}\right)+k_{2} x_{5}+ \\
k_{3}\left(g-\frac{k x_{6}^{2}}{m x_{4}^{2}}+\frac{k_{r}\left(x_{1}-x_{4}\right)}{m}\right)
\end{array}\right]
\end{aligned}
$$

Equations (48) and (49) are the control law applied to the physical controllers.

\section{SIMULATION AND EXPERIMENTS}

For one application system, the parameters of the two subsystems described in Fig. 1 are totally the same.

$$
\begin{aligned}
& k=0.00545, m=725 \mathrm{~kg} \\
& s_{0}=0.012 \mathrm{~m}, R=4.44 \Omega \\
& g=9.8 \mathrm{~N} / \mathrm{kg}, k_{r}=1.236 \times 10^{7} \mathrm{~N} / \mathrm{m}
\end{aligned}
$$

When the power of one controller of the joint-structure is suddenly shut down, the simulation results of the other controller are shown in Fig. 2.

When the power of one controller of the joint-structure is suddenly shut down, the experimental results of the other controller are shown in Fig. 3.

From simulation results shown in Fig. 2, it can be seen that when one controller breaks down, the current for the other controller increase rapidly from $18 \mathrm{~A}$ to $24 \mathrm{~A}$, and the maximum variety of the suspension gap is $0.5 \mathrm{~mm}$.

From experimental results shown in Fig. 3, it can be seen that when one controller breaks down, the current for the other controller increase rapidly from $17 \mathrm{~A}$ to $25 \mathrm{~A}$, and the maximum variety of the suspension gap is $1.6 \mathrm{~mm}$. And the suspension gap finally reaches the expected value because of the effect of integrator. 

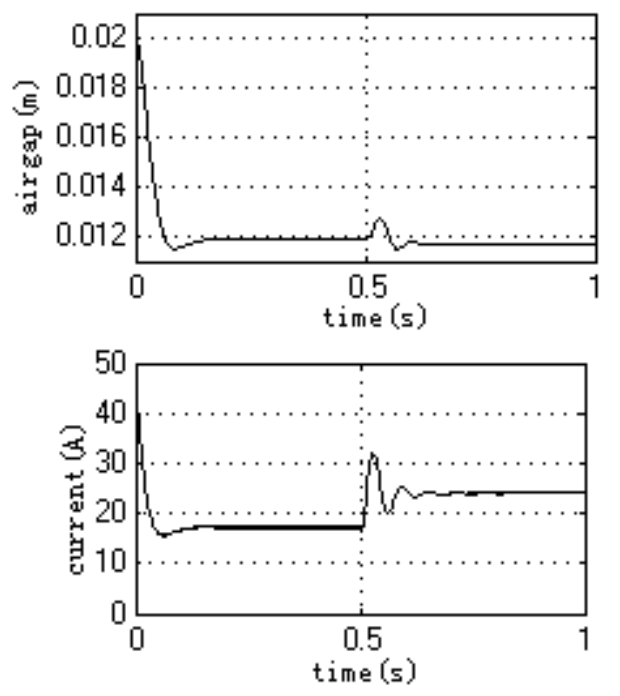

Fig. 2: Simulation result of air-gap and current

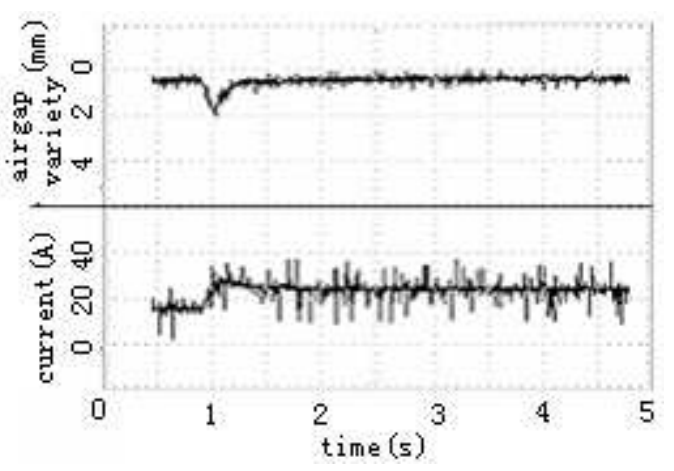

Fig. 3: Experiment results of air-gap variety and current

\section{CONCLUSIONS}

The nonlinear model for joint-structure of maglev vehicles are built in this paper. And the obtained model is a multiple-inputs multiple-outputs coupling model. Nonlinear state feedback technique is introduced to decouple and linearize this model. Then pole assignment is utilized to design the controller. The proposed control algorithm is simple, and convenient to implement.

The control strategy proposed in this paper is validated by both simulation and experiment. Simulation and experimental results show that, both the two controllers for a single joint-structure can realize stable suspension, which means the proposed method is effective for decoupling control. And it can be also shown that, when one controller breaks down, the other controller can realize the stability of the whole joint-structure system by itself. Joint-structure function is effectively realized by the proposed method, which can improve the safety of maglev vehicles.
The authors are grateful to the anonymous referee for a careful checking of the details and for helpful comments that improved this paper.

\section{References}

[1] S. J. Joo and J. H. Seo. Design and analysis of the nonlinear feedback linearizing control for an electromagnetic suspension system [J]. IEEE Trans. Contr. Syst. Technol, 5, 135-144 (1997).

[2] R.J.kavanagh.Multivarible control system synthesis [J]. AIEE.Trans Apr Ind., 77, 425-429 (1988).

[3] K Weischedel, T J Mcavoy. Feasiblity of decoupling in conventionally controlled distillation columns[J]. Ind Eng Chen, 19, 167-172 (1990).

[4] F G Shiskoy.The stability of interacting control loops with and without Decoupling [J]. Proc IFAC multivariable Technological System Cont 4th International Symposium, 121-134 (1977).

[5] H H Rosebrocok. Design of Multivariable Control System using Inverse Nyquist Array [J]. Pro IEEE, 1916-1929 (1969).

[6] Elmer G Gilbert. The Decoupling of Multivariable Systems by State Feedback [J]. SIAM.J Control, 221-229 (1989).

[7] Narendra K and Parthasarathy K. Identification and control of dynamic systems using neural networks IEEE Trans [J]. On Neural Networks, 1, 4-27 (1990).

[8] Henriquest $\mathbf{J}$ and Dourado A. A Multivariable adaptive control using a recurrent neural network Proceedings of Eann98[J]. Engineering Applications of Neural Networks, Gibraltar, 9, 118-121 (1998).

[9] Morari,M. Advances in Model Predictive Control Conference [M]. Oxford University Press, 22-27 (1994).

[10] Shaw A and Doyle F. Multivariable non-linear control applications for a high purity distillation column using a recurrent dynamic neuron model $[\mathrm{J}]$. Journal of Process Control, 7, 255-268 (1997).

[11] R Y Chiang, M G Safonov, K Haiges, K Madden and J Tekawy. A fixed $H_{\infty}$ controller for a super maneuverable fighter performing the Herbst maneuver [J]. Automatica, 29, 111-127 (1993).

[12] W Chan, R J Anderson, P Beame, D H Jones, D Notkin and W E Warner. Decoupling Synchronization from Local Control for Efficient Symbolic Model Checking of State Charts [J]. In Proceedings of the 1999 International Conference on Software Engineering (ICSE99), 142-151 (1999).

[13] HengKun Liu. Research on Suspension Control Problems of EMS High-Speed Maglev Train Double Bogies JoinStructure [R]. Doctor dissertation of National University of Defense Technology, (2005).

[14] XiaoHua X., Weibing G. Nonlinear control and decoupling [M], Science Publisher, (1993).

[15] Ling W. Automatic control principle [M]. Tinghua University Publisher, (1990). 

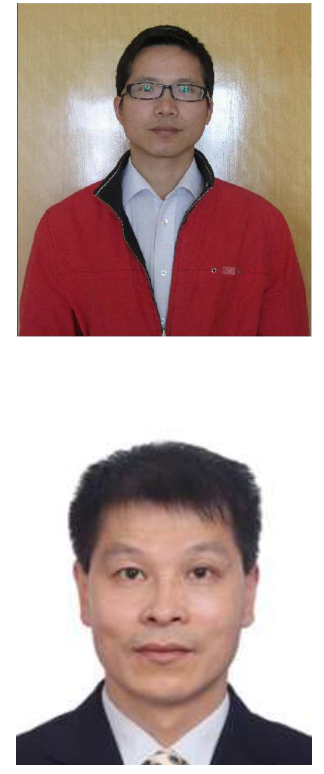

levitation control technique.
Hengkun Liu was born in Jangjin, Chongqing, China, in 1975. He received the PH.D degree in 2005works for National University of Defense Technology as a teacher. His research interests include control theory and its application, and maglev train levitation control technique.

Yungang Li was born in Tianmen county, Hubei Province, China, in 1969. He received the PH.D degree in 1996works for National University of Defense Technology as a Professor. His research interests include control theory and its application, and maglev train

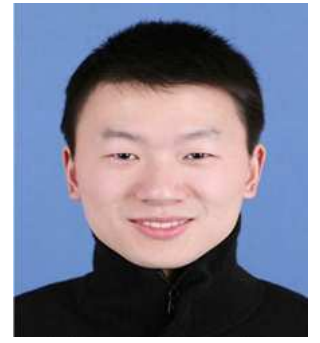

Hu Cheng was born in Xiangfan county, Hubei Province, China, in 1981. $\mathrm{He}$ received the PH.D degree in 2011works for National University of Defense Technology as a teacher. His research interests include control theory and its application, and maglev train

levitation control technique. 